\title{
Revascularization in patients with heart failure
}

\author{
Ross T. Tsuyuki, Fiona M. Shrive, P. Diane Galbraith, Merril L. Knudtson, Michelle M. Graham, \\ for the APPROACH Investigators
}

$\infty \quad$ See related article page 372

\section{ABSTRACT}

Background: Although practice guidelines recommend coronary revascularization for patients with heart failure, the evidence to support this recommendation is weak. The objective of our study was to determine the association of coronary revascularization with survival in patients who have had heart failure.

Methods: Data were obtained from the Alberta Provincial Project for Outcomes Assessment in Coronary Heart Disease (APPROACH), a clinical outcome-monitoring initiative that has captured data on all patients undergoing cardiac catheterization in the province of Alberta since 1995. Our study included data from patients with a history of heart failure and with documented coronary artery disease; patients with normal coronary arteries or prior coronary artery bypass grafting (CABG) were excluded. We constructed survival curves and adjusted them by the corrected group prognosis method (incorporating all clinical variables in APPROACH). Propensity scores were used to account for clinical characteristics that could influence the decision to revascularize.

Results: A total of $253^{8}$ patients (mean age $68 \mathrm{yr}$, standard deviation [SD] $11 \mathrm{yr}, 31 \%$ female) underwent revascularization; 1690 patients (mean age 69 [SD 11] yr, 34\% female) did not. Crude 1 -year mortality was $11.8 \%$ among patients who underwent revascularization, compared with $21.6 \%$ among those who did not. Adjusted survival curves diverged early and continued up to 7 years of follow-up (hazard ratio 0.50 , $95 \%$ confidence interval $0.44-0.57$ ). Propensity scores showed improved survival with revascularization across all quintiles of likelihood of revascularization.

Interpretation: This new evidence lends support to practice guidelines, which recommend revascularization in patients with heart failure and coronary disease.

CMAJ 2006;175(4):36I-5 lthough the prevalence of cardiovascular disease is generally on the decrease in Western society, that of heart failure is, paradoxically, increasing, owing to improved survival of patients with cardiovascular disease and a generally aging population. ${ }^{1-4}$ Heart failure is associated with very high rates of illness and death, and constitutes an important public health problem. In Canada in the year 2000, heart failure accounted for over 106 ooo hospital admissions and I 400000 inpatient days, which reveals it to be one of the most prevalent acute-care diagnoses. ${ }^{2}$

Coronary artery disease is the primary etiology in twothirds of all cases of heart failure. ${ }^{4}$ In addition, uncontrolled coronary ischemia has been identified as a common precipitant of heart-failure exacerbations. ${ }^{5-7}$ As such, practice guidelines recommend coronary revascularization for patients with heart failure and coronary artery disease. ${ }^{3,4}$

There is, however, relatively little evidence for a strategy of routine revascularization in patients with heart failure, with the only published literature examining coronary-artery bypass grafting (CABG), either from cohort trials or subgroups from randomized trials. ${ }^{8,9}$ To date, no randomized studies of revascularization have been completed that specifically involve patients with heart failure.

We sought to determine the association of coronary revascularization with survival in patients with heart failure, with use of a population-based registry. Our secondary objective was to determine differences in survival based upon revascularization strategy: CABG versus percutaneous coronary intervention (PCI).

\section{Methods}

Data were obtained from a prospective clinical data-collection initiative, the Alberta Provincial Project for Outcomes Assessment in Coronary Heart Disease (APPROACH), which has captured data on all patients who have undergone cardiac catheterization in the province since $1995 .{ }^{10}$ The database contains detailed information including patients' age, sex, left-ventricular ejection fraction, coronary anatomy and comorbidities. It tracks therapeutic interventions such as previous thrombolytic therapy and previous or subsequent revascularization procedures (CABG or PCI). Follow-up mortality is ascertained by means of a semiannual merge with data from the Alberta Bureau of Vital Statistics. This database therefore allows for the study of processes and outcomes of cardiac procedures at the population level.

We included patients with a documented history of heart failure at the time of coronary catheterization. Excluded were patients with normal coronary arteries or nonsignificant coro- 
nary disease (defined as stenoses $<50 \%$ ) and those with prior CABG, because such patients would be unlikely to be recommended coronary revascularization. Cases were then grouped according to whether the patients had undergone revascularization within the first year after catheterization or not. Survival data were collected over the next I-7 years of follow-up.

The APPROACH study protocol was approved by the research ethics boards of the Universities of Calgary and Alberta. The requirement for informed consent was waived.

Patient characteristics among the 2 groups were compared with $\chi^{2}$ tests. Kaplan-Meier plots and log-rank tests were used to determine and compare crude survival rates per age group according to treatment strategy (revascularization or no revascularization). Time to events for survival analyses was measured from the index catheterization.

To address concerns over the prognostic role of clinical factors that simultaneously influence outcomes and the decision to undergo revascularization, we conducted a propensity score analysis as described by Rubin. ${ }^{11} \mathrm{~A}$ logistic regression model was constructed that estimated the probability (i.e., the propensity) of being revascularized based on the clinical characteristics captured in the APPROACH study. The overall study population was then subdivided into quintiles according to propensity for being revascularized. Each of these

Table 1: Demographic and medical characteristics of patients with heart failure* who underwent coronary catheterization, 1995 through 2000 , in \%†

\begin{tabular}{|lrrrr}
\hline $\begin{array}{l}\text { Characteristic } \\
\text { or risk factor }\end{array}$ & $\begin{array}{c}\text { All } \\
n=4228\end{array}$ & No revasc & Revasc & $p$ \\
\hline vge, yr & 68.2 & 68.9 & 67.7 & 0.002 \\
\hline Standard deviation, yr & 10.7 & 10.8 & 10.7 & 0.06 \\
\hline Male & 67.6 & 66.0 & 68.8 & 0.06 \\
\hline Diabetes mellitus & 33.0 & 35.3 & 31.6 & 0.01 \\
\hline Hyperlipidemia & 37.9 & 33.9 & 40.5 & $<0.001$ \\
\hline Hypertension & 59.5 & 59.7 & 59.4 & 0.83 \\
\hline Present smoker & 26.0 & 25.0 & 26.7 & 0.22 \\
\hline Previous smoker & 46.5 & 45.7 & 47.0 & 0.38 \\
\hline Prior cardiovascular event & & & & \\
\hline Myocardial infarction & 74.2 & 69.8 & 77.1 & $<0.001$ \\
\hline Thrombolytic therapy & 13.0 & 8.4 & 16.0 & $<0.001$ \\
\hline PCl & 8.0 & 6.8 & 8.8 & 0.01 \\
\hline Cerebrovascular disease & 11.7 & 11.5 & 11.9 & 0.68 \\
\hline Peripheral-arterial disease & 12.5 & 12.7 & 12.4 & 0.77 \\
\hline COPD & 22.2 & 23.5 & 21.4 & 0.10 \\
\hline Liver or Gl disease & 6.4 & 5.9 & 6.7 & 0.27 \\
\hline Malignancy & 5.6 & 6.2 & 5.2 & 0.16 \\
\hline Renal disease & 9.1 & 8.9 & 9.3 & 0.67 \\
\hline Requires dialysis & 3.4 & 3.6 & 3.2 & 0.50 \\
\hline
\end{tabular}

Note: revasc $=$ revascularization, $\mathrm{PCI}=$ percutaneous coronary intervention, $\mathrm{COPD}=$ chronic obstructive pulmonary disease, $\mathrm{GI}=$ gastrointestinal.

*Excluding patients with normal anatomy and those who previously underwent coronary-artery bypass grafting.

†Except as indicated for age. "propensity groups" contains patients who were and were not revascularized. One-year mortality rates in each of these groups were determined and compared for revascularization versus nonrevascularization subsets.

We constructed survival curves were and adjusted them for risk with the corrected group prognosis method, ${ }^{12}$ incorpor-

Table 2: Clinical characteristics of patients with heart failure* who underwent coronary catheterization, 1995-2000, in \%

\begin{tabular}{|c|c|c|c|c|}
\hline Condition or medication & $\begin{array}{c}\text { All } \\
n=4228\end{array}$ & $\begin{array}{c}\text { No revasc } \\
n=1690\end{array}$ & $\begin{array}{c}\text { Revasc } \\
n=2538\end{array}$ & $\begin{array}{c}p \\
\text { value }\end{array}$ \\
\hline \multicolumn{4}{|c|}{ Primary indication for catheterization } & $<0.001$ \\
\hline Stable angina & 10.0 & 12.1 & 8.5 & \\
\hline Unstable angina & 21.3 & 20.1 & 22.1 & \\
\hline Myocardial infarction & 47.2 & 36.5 & 54.3 & \\
\hline Heart failure & 12.3 & 19.3 & 7.7 & \\
\hline Other† & 9.2 & 12.0 & 7.3 & \\
\hline Coronary anatomy & & & & $<0.001$ \\
\hline Low riskł & 38.0 & 40.5 & 36.4 & \\
\hline High risk $\neq$ & 45.8 & 44.3 & 46.8 & \\
\hline Left main coronary artery & 13.2 & 11.1 & 16.2 & \\
\hline Data unavailable & 2.0 & 4.1 & 0.6 & \\
\hline \multicolumn{4}{|c|}{ Left-ventricular ejection fraction } & $<0.001$ \\
\hline$>50 \%$ & 23.0 & 17.8 & 26.5 & \\
\hline $35 \%-50 \%$ & 30.1 & 26.9 & 32.3 & \\
\hline $20 \%-34 \%$ & 20.7 & 25.9 & 17.3 & \\
\hline$<20 \%$ & 4.4 & 7.2 & 2.5 & \\
\hline Could not be calculated & 0.3 & 0.4 & 0.2 & \\
\hline Not measured & 11.1 & 10.8 & 11.3 & \\
\hline Datum missing & 10.5 & 11.4 & 9.9 & \\
\hline \multicolumn{5}{|l|}{ Medications in use§ } \\
\hline Aspirin & 60.0 & 54.5 & 63.8 & $<0.001$ \\
\hline Ticlopidine/clopidogrel & 1.2 & 0.9 & 1.3 & $<0.001$ \\
\hline ACE inhibitors & 42.9 & 46.5 & 40.5 & $<0.001$ \\
\hline Beta-blocker & 38.2 & 32.9 & 41.7 & $<0.001$ \\
\hline Calcium-channel blockers & 12.8 & 12.3 & 13.0 & 0.001 \\
\hline Glyburide & 3.3 & 3.9 & 3.0 & $<0.001$ \\
\hline Lipid-lowering agents & 13.1 & 12.1 & 13.7 & $<0.001$ \\
\hline Long-acting nitrate & 31.3 & 31.2 & 31.4 & $<0.001$ \\
\hline \multicolumn{5}{|c|}{ Crude mortality rates (total deaths) } \\
\hline At 30 days $(n=267)$ & 6.9 & 8.6 & 5.7 & 0.003 \\
\hline At 1 year $\quad(n=613)$ & 15.8 & 21.6 & 11.8 & $<0.001$ \\
\hline
\end{tabular}

Note: revasc $=$ revascularization, ACE = angiotensin-converting enzyme.

*Excluding patients with normal anatomy and those who previously underwent coronary-artery bypass grafting.

†Serious arrhythmia, nonischemic cardiomyopathy, valvular heart disease or congenital heart disease.

¥Adapted from the Duke classification:

- Low risk: single-vessel disease with $\geq 50 \%$ stenosis or 2 -vessel disease, with or without stenosis (excepting $95 \%$ stenosis in the proximal left anterior descending artery).

- High risk: 2-vessel disease with 95\% stenosis of the proximal left anterior descending artery or 3-vessel disease with or without stenosis.

$\S A t$ the time of cardiac catherization. 
ating all clinical variables collected (Table I and Table 2), excluding medications (listed in Table 2).

Because our data were hierarchical in nature, with data from 4 hospitals and potential within-hospital clustering of data, we carried out sensitivity analyses with generalized estimating equations (proc GENMOD in SAS) as well as a generalized hierarchical model (GLIMMIX macro in SAS) with a random effect for hospital. These analyses changed neither the statistical significance of any of our findings nor the width of our confidence intervals (CIs) in any notable way. We therefore concluded that there was minimal (or no) clustering in our data and that our proportional hazard models were appropriate.

\section{Results}

Of the 6ri34 patients with heart failure who underwent coronary catheterization from I 995 through 200I, I353 were excluded because their coronary arteries were normal, and a further 553 because of prior CABG. A total of 4228 patients therefore met the inclusion criteria, with 2538 receiving revascularization and 1690 patients not receiving it. Demographic and clinical characteristics of these patients are shown in Table $\mathrm{I}$ and Table 2.

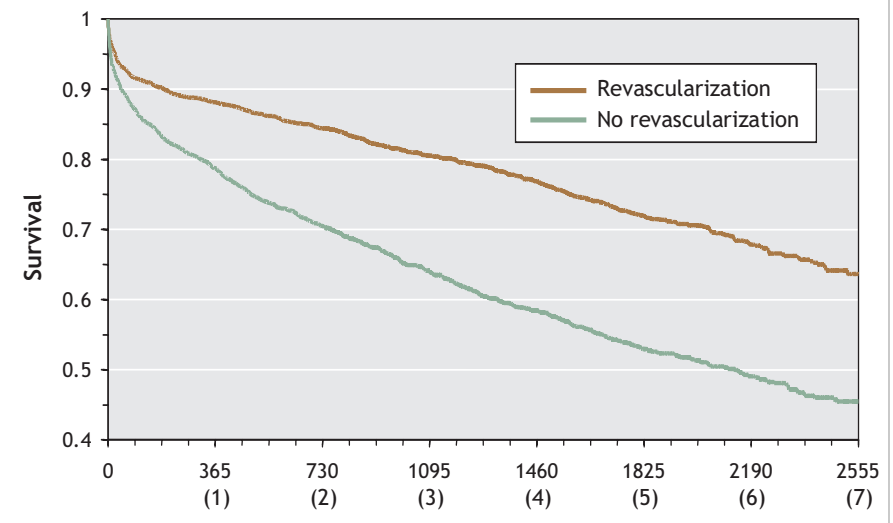

Days (years) from catheterization

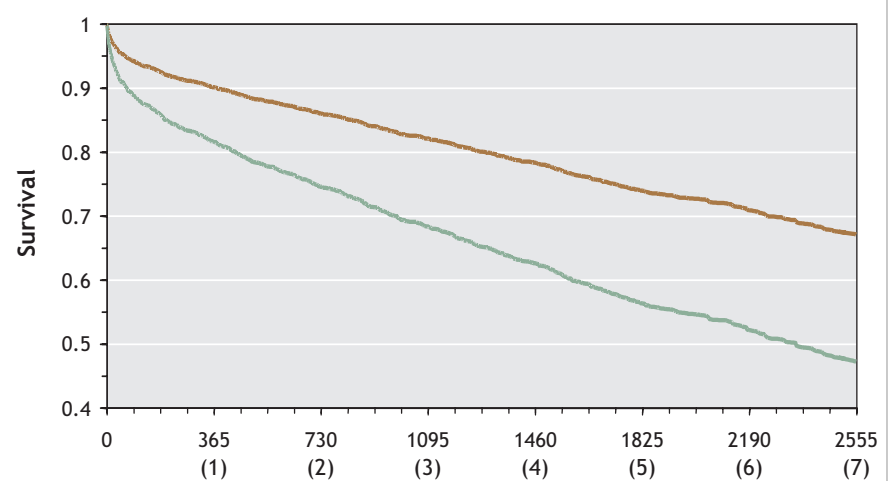

Days (years) from catheterization

Fig 1: Crude survival of patients with heart failure who underwent revascularization compared to those not revascularized. Upper panel, crude survival; lower panel, risk-adjusted survival.
The average age of the patients studied was 68 years; twothirds of them were male. Overall, $60 \%$ of the patients received revascularization, $52.5 \%$ by CABG and $48.5 \%$ by PCI. As expected in this patient population, coronary risk factors were common, the most frequent being hypertension, hyperlipidemia, diabetes and current smoking. Three-quarters of the patients had a prior myocardial infarction. Comorbidities, including chronic obstructive pulmonary, peripheral arterial, cerebrovascular and renal disease, were often present.

The main indications for coronary catheterization were myocardial infarction $(47 \%)$, unstable angina $(2 \mathrm{I} \%)$ and heart failure ( $12 \%)$. Most patients had left-ventricular ejection fractions of less than $50 \%$. In general, patients who were revascularized were slightly younger, were more likely to have had a prior myocardial infarction, had a higher ejection fraction, and were more likely to have high-risk coronary anatomy (2vessel disease with involvement of the proximal left anterior descending artery, 3-vessel disease or left main coronaryartery disease). Medications at the time of catheterization included ASA in $60 \%$, angiotensin-converting enzyme (ACE) inhibitors in $43 \%$, $\beta$-blockers in $38 \%$, long-acting nitrates in $31 \%$, calcium-channel blockers in $13 \%$ and lipid-lowering agents in $13 \%$.

Crude survival curves for the patients who underwent revascularization and those who did not are shown in the upper panel of Fig. I. Corresponding mortality rates at I year were II. $8 \%$ in those who underwent revascularization and $21.6 \%$ in those who did not (hazard ratio [HR] 0.52, 95\% CI 0.47$0.58)$. The risk-adjusted 7-year survival curves, meanwhile (Fig. I, lower panel), diverged early and continued diverging in favour of revascularization for up to 7 years of follow-up (HR 0.50, 95\% CI 0.44-0.57). Propensity-adjusted relative survival is shown in Table 3 . Within all 5 propensity quintiles of likelihood of revascularization, patients who received revascularization had an improved survival, with a relative risk ranging from $\mathrm{I} .79$ to 2.86 .

Crude and adjusted 7-year survival curves are shown in Fig. 2, comparing patients who underwent PCI or CABG with those who did not. Based upon the crude survival curve (upper panel), CABG and PCI appear to confer similar survival benefits (CABG HR 0.5I, 95\% CI 0.44-0.59; PCI HR 0.46, 95\% CI 0.39-0.54 [each v. no revascularization]). The adjusted curve (Fig. 2, lower panel) shows CABG to be associated

Table 3: Propensity-adjusted relative survival, scored from 1 (least likely to be revascularized) through 5 (most likely)

\begin{tabular}{|c|c|c|c|c|c|}
\hline \multirow{2}{*}{$\begin{array}{l}\text { Score } \\
\text { quintile }\end{array}$} & \multicolumn{2}{|c|}{ No. of patients } & \multicolumn{3}{|c|}{ Survival at 1 year, \% } \\
\hline & No revasc & Revasc & No revasc & Revasc & RR (95\% Cl) \\
\hline 1 & 421 & 230 & 79.8 & 89.6 & $1.92(1.3-2.9)$ \\
\hline 2 & 366 & 297 & 80.1 & 88.8 & $1.79(1.2-2.6)$ \\
\hline 3 & 260 & 405 & 80.0 & 89.1 & $1.85(1.3-2.6)$ \\
\hline 4 & 191 & 454 & 82.2 & 90.5 & $1.89(1.2-2.9)$ \\
\hline 5 & 137 & 539 & 79.6 & 92.8 & $2.86(1.8-4.3)$ \\
\hline
\end{tabular}

Note: revasc = revascularization, $\mathrm{RR}=$ relative risk, $\mathrm{Cl}=$ confidence interval. 
with the best survival (HR 0.44 v. no revascularization, 95\%

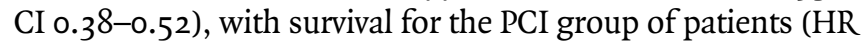
0.58 v. no revascularization, $95 \% \mathrm{CI} 0.49^{-0.69)}$ lying between that of the $\mathrm{CABG}$ and no-revascularization groups.

\section{Interpretation}

Coronary artery disease is highly relevant to the management of patients with heart failure, as it represents the single most important cause of heart failure and is a major factor in exacerbations of symptoms. ${ }^{1,3-7}$ Therefore, examination is needed of the role that coronary revascularization plays in the management of patients with heart failure. In our analysis of a large cohort of patients with heart failure who underwent cardiac catheterization, we demonstrated a strong association between revascularization and survival. This finding provides valuable support to the practice guidelines for heart failure.

All recent practice guidelines recommending coronary revascularization in patients with heart failure cite the systematic review conducted by Baker and associates in $1994 .{ }^{8}$ The authors evaluated 8 cohort studies that involved 2695 patients whose heart failure was treated with CABG or medical ther-

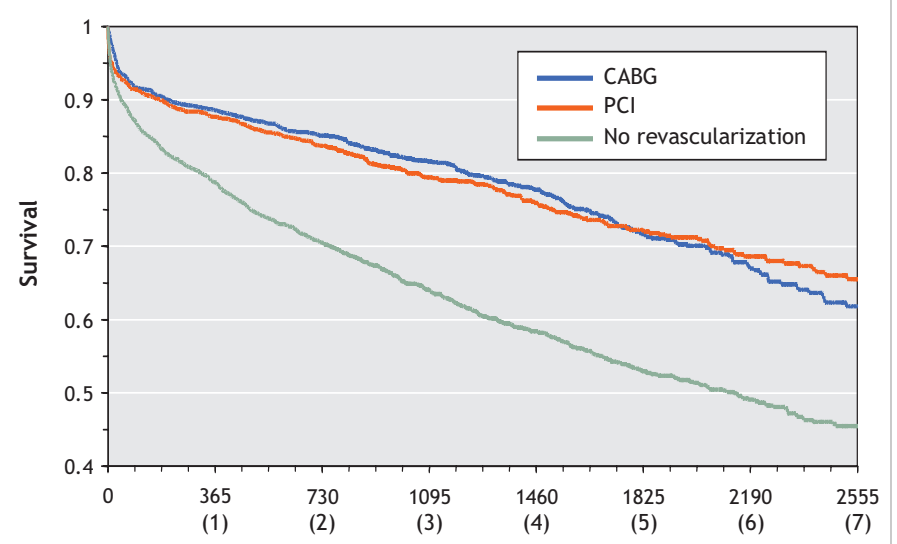

Days (years) from catheterization

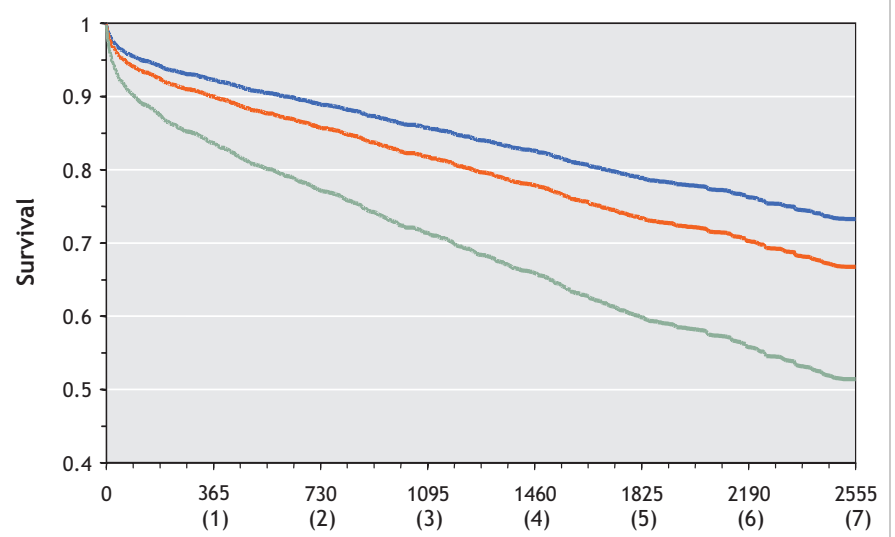

Days (years) from catheterization

Fig 2: Survival of patients with heart failure, according to revascularization strategy. Upper panel, crude survival; lower panel, risk-adjusted survival. apy. The studies' results were not combined for meta-analysis because of a high degree of heterogeneity. No studies of PCI were included, because none were available when that review was conducted. Baker's group concluded that, in patients with heart failure and limiting angina, CABG improved 3-year survival by $30 \%-50 \%$ and physical function by about $1 \mathrm{New}$ York Heart Association Functional class. Although they used quality ratings for cohort studies, the causal inference from such study designs is rather limited. In a systematic review by Yusuf and coworkers ${ }^{9}$ of 2649 patients randomized to CABG or to medical treatment, it was noted that the subgroup of patients with lower ejection fractions had relative benefits of CABG similar to those with ejection fractions in the normal range. However, because of their higher mortality risk, patients with lower ejection fractions had a greater absolute benefit from CABG.

Our study, using a truly population-based cohort of patients with heart failure who were referred for cardiac catheterization, combined with powerful statistical techniques, observed a striking association with improved survival in patients undergoing revascularization. Although these are observational data, with a lower level of causal inference than a randomized trial, this may be the best available evidence to-date for the efficacy of revascularization in patients with heart failure. Our results suggest that all patients with heart failure and clinically significant coronary artery disease amenable to revascularization should be considered for these procedures.

Our secondary objective was to evaluate differences between PCI and CABG in the survival of patients with heart failure. In the adjusted analysis (Fig. 2, lower panel) patients undergoing $C A B G$ appeared to have slightly better survival than those who received PCI. The results of these subgroup analyses should be viewed with caution, however: the complexity of clinical decision-making for revascularization is likely not completely accounted for, in our adjustments. In any case, differences in survival by revascularization type are modest compared with those between patients who underwent revascularization and those who did not.

It is also notable that less than half of the patients were receiving ACE inhibitors or $\beta$-blockers at the time of their coronary catheterization. Although about a quarter of the patients had preserved systolic function, these observations are consistent with other reports of the underuse of the therapies proven efficacious in heart failure. ${ }^{13,14}$ It can be argued that the survival rate of nonrevascularized patients is perhaps not reflective of what might be seen in a nonrevascularized cohort that receives optimal medical therapy.

The causal inference that can be drawn from an observational study, no matter how well conducted or analyzed, will always be less robust than that drawn from an appropriately powered randomized controlled trial. There may be clinical details that influenced the decision to revascularize that were not captured. Nevertheless, the propensity score analysis indicates that the study findings are robust and consistent across groups of patients with different probabilities for selection for revascularization. Second, the patient population evaluated in our study was confined to those who were referred for cardiac catheterization. We recognized that this 
would not include all patients with heart failure, and that we would therefore be unable to determine if a strategy of routine revascularization would be beneficial to patients with heart failure and asymptomatic or minimally symptomatic coronary artery disease. To protect the internal validity of our study, we excluded patients with normal coronary arteries or coronary disease that would not be clinically significant (i.e., those who would not need revascularization). Third, we did not apply strict diagnostic criteria for heart failure in this population. "Heart failure" was a clinical diagnosis, documented by the referring physicians. Nevertheless, the ejection fraction was low in most patients, suggesting that these assumptions were applicable. Fourth, we did not have information on the use of implantable cardioverter-defibrillator devices. Finally, we only had data on the medications being taken at the time of the procedure. Postprocedure medications may be important covariates, if their use differed between groups.

Given the implications of heart failure for public health, a randomized trial of revascularization for patients with heart failure is needed. Currently, the Surgical Treatment for Ischemic Heart Failure (STICH) randomized multicentre trial is evaluating medial therapy versus CABG surgery for patients with heart failure, an ejection fraction of less than $35 \%$ and coronary artery disease suitable for revascularization. ${ }^{15}$ The trial, which began in July 2002, aims to enrol 2800 patients at 50 sites in North America and Europe, with follow-up of at least 3 years. In contrast to our study, STICH will exclude patients who are candidates for PCI. This trial is scheduled for completion in 2008; until then, the best available evidence must be drawn from observational studies.

In this analysis of a large cohort of patients with heart failure who underwent catheterization, a strong association was revealed between revascularization and improved survival. This finding supports current recommendations in practice guidelines on heart failure and suggests that revascularization should be considered for all patients with heart failure and coronary artery disease.

This article has been peer reviewed.

From the Division of Cardiology, University of Alberta (Tsuyuki, Graham), Edmonton, and University of Calgary (Shrive, Galbraith, Knudtson), Calgary, Alta.

Competing interests: None declared.

Contributors: Ross Tsuyuki conceived and designed the project, participated in the interpretation of the data and drafted the manuscript. Fiona Shrive and P. Diane Galbraith shared responsibility for data acquisition. P. Diane Galbraith, Merril Knudtson and Michelle Graham contributed to study design and data interpretation; Merril Knudtson is the overall principal investigator for the APPROACH project. All coauthors participated in the critical revision of the article and approved the final version to be published.
Acknowledgements: The APPROACH Steering Committee includes Stephen Archer, Micheal Curtis, William A. Ghali, Michelle Graham, William Hui, Merril L. Knudtson, Arvind Koshal, Andrew Maitland, L. Brent Mitchell and Ross T. Tsuyuki (Chair).

APPROACH was initially funded with a grant from the W. Garfield Weston Foundation, and is ongoing because of operating grants from the Heart and Stroke Foundations of Alberta, Northwest Territories and Nunavut, and the Canadian Institutes of Health Research (CIHR). The initiative also has received contributions from Alberta Health and Wellness, Merck Frosst Canada Inc., Monsanto Canada Inc.-Searle, Eli Lilly Canada Inc., Guidant Corporation, Boston Scientific Ltd., Hoffmann-La Roche Ltd. and Johnson \& Johnson Inc.-Cordis.

Ross Tsuyuki holds the Merck Frosst Chair in Patient Health Management from the University of Alberta. Diane Galbraith is supported by a Canadian Cardiovascular Outcomes Research Team student fellowship, funded by an operating grant from CIHR and the Heart and Stroke Foundation, and a TORCH (Tomorrow's Research Cardiovascular Health Professionals) student fellowship. Merril Knudtson receives partial support from the Libin Trust Fund.

\section{REFERENCES}

I. Jessup M, Brozema S. Heart Failure. N Engl JMed 2003;348:2007-I8.

2. Tsuyuki RT, Shibata MC, Nilsson C, et al. Contemporary burden of illness of congestive heart failure in Canada. Can J Cardiol 2003;19:436-8.

3. Liu P, Arnold M, Belenkie I, et al. The 200I Canadian Cardiovascular Society consensus guideline update for the management and prevention of heart failure. Can Cardiol 200I; I7(Suppl E):5E-24E.

4. Hunt SA, Baker DW, Chin MH, et al. ACC/AHA guidelines for the evaluation and management of chronic heart failure in the adult: a report of the American College of Cardiology/American Heart Association Task Force on Practice Guidelines (Committee to Revise the 1995 Guidelines for the Evaluation and Management of Heart Failure. J Am Coll Cardiol 200I;38:210I-I3.

5. Chin MH, Goldman L. Factors contributing to the hospitalization of patients with congestive heart failure. Am J Public Health $1997 ; 87: 643-8$.

6. Opasich C, Febo O, Riccardi G, et al. Concomitant factors of decompensation in chronic heart failure. Am J Cardiol 1996;78:354-7.

7. Ghali JK, Kadakia S, Cooper R, et al. Precipitating factors leading to decompensation of heart failure. Arch Intern Med I988;148:2013-6.

8. Baker DW, Jones R, Hodges J, et al. Management of heart failure. III. The role of revascularization in the treatment of patients with moderate or severe left ventricular systolic dysfunction. JAMA I994;272:1528-34.

9. Yusuf S, Zucker D. Effect of coronary bypass graft surgery on survival: overview of Io-year results from randomized trials by the Coronary Artery Bypass Graft Surgery Trialists Collaboration. Lancet 1994;344:563-70.

Io. Ghali WA, Knudtson ML. Overview of the Alberta Provincial Project for Outcome Assessment in Coronary Heart Disease. Can J Cardiol 2000;16:1225-30.

II. Rubin DB. Estimating causal effects from large data sets using propensity scores. Ann Intern Med I997; 127:757-63.

I2. Ghali WA, Quan H, Brant R, et al. Comparison of 2 methods for calculating adjusted survival curves from proportional hazards models. JAMA 2001;286:1494-7.

I3. Clinical Quality Improvement Network Investigators. Mortality risk and patterns of practice in 4606 acute care patients with congestive heart failure: the relative importance of age, sex, and medical therapy. Arch Intern Med 1996;156:1669-73.

I4. Bungard TJ, Johnson JA, McAlister FA, et al. Underutilisation of ACE inhibitors in patients with congestive heart failure. Drugs 200I;6I:202I-33.

15. Joyce D, Leobe M, Noon GP, et al. Revascularization and ventricular restoration in patients with ischemic heart failure: the STICH trial. Curr Opin Cardiol 2003; I8:454-7.

Correspondence to: Dr. Michelle M. Graham, Division of Cardiology, 2C2 Walter Mackenzie Centre, University of Alberta Hospital, 8440 - II2 St., Edmonton AB T6G 2B7;

mmgraham@cha.ab.ca 